LAAUR $9 \%=601$

Title:

\title{
ADVANCED TECHNIQUES FOR THE ANALYSIS OF CRISIS STABILITY, DETERENCE, AND LATENCY
}

Author(s):

Submitted to:

Gregory H. Canavan, P-DO

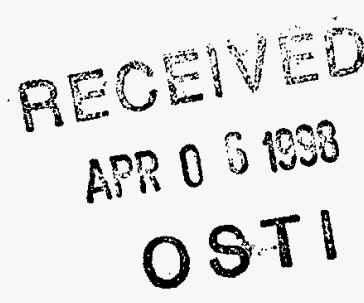

DISTRBSUTION OF THIS DOCUMENT IS UNLIMTTED

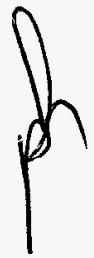

For discussions outside the Laboratory

Date: December 1997

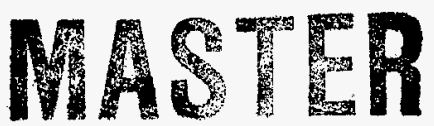

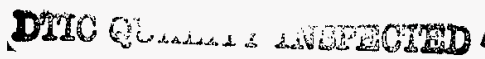

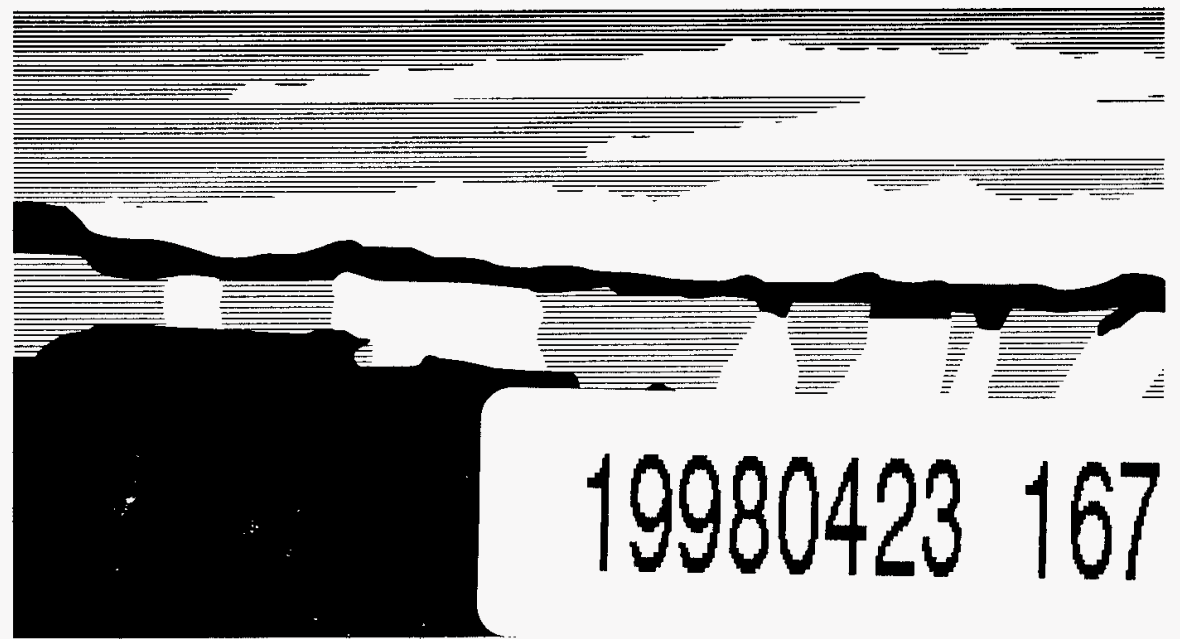

NATIONAL LABORATORY

Los Alamos National Laboratory, an affirmative action/equal opportunity employer, is operated by the University of California for the U.S. Department of Energy under contract W-7405-ENG-36. By acceptance of this article, the publisher recognizes that the U.S. Government retains a nonexclusive, royalty-free license to publish or reproduce the published form of this contribution, or to allow others to do so, for U.S. Government purposes. The Los Alamos National Laboratory requests that the publisher identify this article as work performed under the auspices of the U.S. Department of Energy. 


\section{DISCLAIMER}

This report was prepared as an account of work sponsored by an agency of the United States Government. Neither the United States Government nor any agency thereof, nor any of their employees, makes any warranty, express or implied, or assumes any legal liability or responsibility for the accuracy, completeness, or usefulness of any information, apparatus, product, or process disclosed, or represents that its use would not infringe privately owned rights. Reference herein to any specific commercial product, process, or service by trade name, trademark, manufacturer, or otherwise does not necessarily constitute or imply its endorsement, recommendation, or favoring by the United States Government or any agency thereof. The views and opinions of authors expressed herein do not necessarily state or reflect those of the United States Government or any agency thereof. 


\title{
ADVANCED TECHNIQUES FOR THE ANALYSIS OF CRISIS STABILITY, DETERRENCE, AND LATENCY
}

\author{
Gregory H. Canavan
}

\begin{abstract}
Studies on crisis stability, deterrence, and latency are presented in chronological order, which also reflects their logical order of development capture the main features of stability analysis; relates first strike, crisis, and arms control stability as seen from U.S. and Russian perspective; and address questions such a whether uncertain in damage preference or defense deployment can be destabilizing. It illustrates the problems with alternative metrics, latency and reconstitution, and deep unilateral and proportional force reductions.
\end{abstract}

Introduction. This report reviews and discusses a series of studies on crisis stability, deterrence, and latency. The discussions of the component reports are presented in chronological order, which also reflects the logical order of the development of the main concepts. The study examined flexible modeling of missile exchanges, optimal allocation of strikes, sensitivity of results to stability metrics, and the effect of the time dependence of nuclear stockpiles at START II levels and beyond. Its major goal was to build time dependence and nuclear latency into a bipolar stability model and explore their impacts at low nuclear force levels, which was met.

Four guides made it possible to efficiently search through the models for a range of missile forces and metrics to produce a fairly simple assessment of the stability properties of this class of models. Using U.S. exchange models and costs and Russian attack optimization made it possible to connect the two analyses and explore reasons for the earlier differences between them. It also provided the first strike cost derivative information with respect to missiles and weapons that defines the boundary between growth or decay of offensive forces. The results require an estimate of the opponent's damage preference, but they provide a simple framework for assessing sensitivity to it and possibly for inferring preferences from past behavior.

Model calculations with various missile and weapon mixes provide a matrix with which it is possible to see essential interrelationships directly. In most cases they can be reduced to simple dimensionless ratios. The basis for and effect of changing allocations then becomes clear, as do the reasons for the intermediate dip in stability as defenses are introduced, the loss of flexibility under unacceptable damage, and the inaccessibility of maximizing stability. The problems and payoffs of proportional or unilateral arms reductions are subject to a fairly simple interpretation, as is their sensitivity to hidden or added weapons.

Time dependence is an important and interesting subject that is treated well by this analysis, although that has not been explored fully. A limited number of calculations explored the sensitivity of missile buildups and decays to a few simple parameters-primarily the damage 
preference. The resulting bifurcations are plausible and in accord with Russian concerns, but need to cover missile and weapon mixes and fiscal costs fully and to be explored with a range of parameters for latency, reconstitution, and added weapons.

Linearity of costs and indices for small forces make it possible to explore the stability characteristics at the goal of current reductions quite thoroughly. It permits exact optimization of attacks, analytic discussion of the role of survivable forces, and derivation of bilinear stability analyses, which clearly delineate the respective roles of missiles and weapons in stability and reconstitution. Since indices reduce to bilinear products of the number of vulnerable missiles and the difference between normalized second and first strikes, there are three ways to produce stability: reduce missiles to zero, reduce $\mathrm{s}-\mathrm{f}$ to zero, or use inaccurate missiles or hidden launchers. It is likely that all three will be needed to reach current objectives.

These models can be applied with little modification to conventional or WMD forces, where they reveal a little-discussed problem. The kill probability of nuclear weapons against conventional and WMD forces degrades rapidly with dispersal, so they are reallocated to value, becoming part of the problem rather than the solution. Conventional and WMD would disperse rapidly, which implies use while they are still effective. These are problems of current interest. Thus, the stability analysis summarized here has been effective in pulling together disparate analyses, useful in providing a road map of the transition ahead, and fruitful in identifying connections with related issues and problems.

\section{Stability at Symmetric Low Force Levels (LA-UR-96-1631, May 1996)} reviews model stability calculations published by members of the Russian General Staff and investigates the basis for their stated concerns over parameters which appear innocuous in US analyses. This note reproduces the results of the Russian model, evaluates exchange ratios, costs, and stability indices within its context, and discusses their sensitivity to the two sides' attack preferences, i.e., their relative preference for inflicting damage on the other relative to that for preventing damage to self.

Those preferences propagate through the Russian stability analyses via their use of attack optimization by minimization of first strike cost. Russian concerns are shown to derive from the recognition that each side knows its own attack preference, but not the other's. In that situation, military prudence dictates worst case planning, i.e., that his opponent is aggressive, induces him to become a reluctant first striker. The logical outcome of this model is that one side strikes first to limit damage and the other side strikes back on the military value targets, i.e., the airbases, ports, etc. that would be used for reconstitution. The key new element of this analysis is that one side's uncertainty about the other side's intentions can convert a nominally stable situation into a strategically unstable one.

2. Elements of Offensive Stability Calculations (LA-UR-96-1632, May 1996) 
further examines the sensitivities identified in the previous note and examines their sensitivity to force, cost, and index parameters. It demonstrates that for symmetric forces and parameters, the attack preference parameter cancels out of the expression for the stability index, but not from the first strike cost that governs the first striker's decision to attack, which is pursued further through the analytic optima derived below.

3. Destabilizing Effects of Perceptions (LA-UR-96-1742, May 1996) explores the instability caused by one side's imperfect knowledge of the other's attack preference for the case of unsymmetric forces, which makes the source of the instability clearer. It shows that if one side assumes the other is not aggressive, he may suffer massive damage if that assumption is incorrect. Conversely, if he assumes the other is aggressive, he may be induced to start an unnecessary exchange. The dilemma is that prudent assessment by either side acting alone cannot produce an outcome as good as that which would result from both sides doing nothing, which is related to the children's' game of prisoners' dilemma. This problem is fundamental, appears at large as well as small force levels, and is exhibited by models with more complicated forces, targets, and indices. Avoiding it appears to require confidence building and information exchange about capabilities and intents that are most sensitive and least likely to be shared.

4. Costs of Strikes between Vulnerable Missile Forces (LA-UR-97-663, Feb 1997) studies symmetric vulnerable missile forces of comparable size and capability with multiple independent reentry vehicles (MIRVs).

The exchange model treats exchanges between symmetric missiles forces in terms of the first, $F$, and second, $S$, strikes either side could deliver. For a force of $M$ missiles with $\mathrm{m}$ weapons each, of which a fraction $f$ are directed at the opponent's $M$ missiles, the first strike on military value targets is

$$
\mathrm{F}=(1-\mathrm{f}) \mathrm{mM} \text {, }
$$

and the average number of weapons delivered on each missile is

$$
\mathrm{r}=\mathrm{fmM} / \mathrm{M}=\mathrm{fm} \text {. }
$$

For $r$ large, the average probability of survival is derived in the following report to be

$$
\mathrm{Q} \approx \mathrm{q}^{\mathrm{r}} \text {, }
$$

where $\mathrm{p}=1-\mathrm{q}$ is the attacking missile's single shot probability of kill. The second strike is

$$
\mathrm{S}=\mathrm{mMQ} \approx \mathrm{mMq}^{\mathrm{r}} \text {, }
$$

delivered on value targets, as missiles remaining at the end of the exchange are taken to have zero value. The report uses these equations, together with numerical optimization of $f$, to examine the stability of the transition from MIRV to singlet vulnerable missiles.

Costs. The first and second strike magnitudes from Eqs. (1) - (4) are converted into costs, measured in terms of the physical damage done to one's opponent and self. The two main objectives in those exchanges are to minimize the damage inflicted on oneself and to retain the 
ability to inflict damaging on the other in order to deter his action. For the former, the relevant cost is the imperfect denial of damage to self. For the latter, the cost is the portion of one's damage objectives one is unable to achieve. Costs are thus measured by the adequacy of delivered first and second strikes relative to cumulative value functions, which are characterized by a parameter $\mathrm{k}$ roughly equal to the inverse of the size of the value target sets held at risk.

The normalized cost to self (s) for the side that strikes first (1) can be approximated by $\mathrm{C}_{1 \mathrm{~s}}=1-\mathrm{e}^{-\mathrm{kS}}$, because the first striker only has to absorb the other's second strike, $\mathrm{S}$. For kS small, $\mathrm{C}_{1 \mathrm{~s}} \approx \mathrm{kS}$ is also small. This form of $\mathrm{C}_{1 \mathrm{~s}}$ is simple, plausible, and exhibits diminishing returns. More detailed forms would involve preferences, priorities, and deterrents that are not known or knowable. The cost of incomplete realization of one's damage objectives (o) can be approximated by $\mathrm{C}_{10}=\mathrm{e}^{-\mathrm{kF}}$, which is small for $\mathrm{kF}$ large and approaches unity for $\mathrm{kF}$ small.

The cost of damage to self and of incomplete damage to the other are incommensurate, as they are fall on different parties; thus, they are formally not an appropriate basis for decisions by either. However, a conventional prescription is to take their weighted sum

$$
\mathrm{C}_{1}=\left(\mathrm{C}_{1 \mathrm{~s}}+\mathrm{LC}_{1 \mathrm{o}}\right) /(1+\mathrm{L})=\left(1-\mathrm{e}^{-\mathrm{kS}}+\mathrm{Le}^{-\mathrm{kF}}\right) /(1+\mathrm{L}),
$$

where $\mathrm{L}$ is a constant. $\mathrm{L}$ small means $\mathrm{C}_{1} \approx \mathrm{C}_{1}$ s, so the first striker is primarily concerned about denying damage to self. $\mathrm{L}$ large means $\mathrm{C}_{1} \approx \mathrm{C}_{1}$, so he is more interested in inflicting damage on the other. It is generally assumed that $0 \leq \mathrm{L} \leq 1$, but that is not required. This construction of $\mathrm{C}_{1}$ as a weighted average of the cost to self and other is plausible, but not unique. However, it reduces all complications to the choice of $\mathrm{L}$, the sensitivity to which is explored below.

Second strike costs are evaluated similarly. The normalized cost to self for the second striker, who must ride out the undiminished first strike $F$, is $C_{2 s}=1-e^{-k F}$, which is $\approx 1$ for $F$ large, when his loss is near total, and $\approx \mathrm{kF}$. for $\mathrm{F} \ll<1 / \mathrm{k}$. The cost of the second striker's partial realization of his damage objectives with his diminished force $S$ is $C_{20}=e^{-k S}$, which is small for $\mathrm{kS}$ large, but $\approx 1$ for $\mathrm{kS} \ll 1$. If these two types of costs are weighted with the same constant $\mathrm{L}$ used above, the total cost of striking second is

$$
\mathrm{C}_{2}=\left(1-\mathrm{e}^{-\mathrm{kF}}+\mathrm{Le}-\mathrm{kS}\right) /(1+\mathrm{L}) \text {. }
$$

The stability metric is taken to be the ratio of the costs for striking first and second. The justification is heuristic. If the cost of striking first, $\mathrm{C}_{1}$, is much smaller than $\mathrm{C}_{2}$, the first striker could see some advantage in attacking, so stability would be reduced. If the cost of striking first is comparable to the cost of striking second, the would-be first striker should see no advantage in initiating an exchange. The ratio $\mathrm{C}_{1} / \mathrm{C}_{2}$ captures both of these influences in a single numerical stability index 


$$
\mathrm{I}=\mathrm{C}_{1} / \mathrm{C}_{2}=\left(1-\mathrm{e}^{-\mathrm{kS}}+\mathrm{Le}-\mathrm{kF}\right) /\left(1-\mathrm{e}^{-\mathrm{kF}}+\mathrm{Le} \mathrm{e}^{-\mathrm{kS}}\right) .
$$

For $\mathrm{kF}$ and $\mathrm{kS}$ small, $\mathrm{I}$ reduces to

$$
\mathrm{I}(\mathrm{F}, \mathrm{S}<<1 / \mathrm{k}) \approx[1-\mathrm{k}(\mathrm{F}-\mathrm{S} / \mathrm{L})] /[1-\mathrm{k}(\mathrm{S}-\mathrm{F} / \mathrm{L})] .
$$

For $L$ large, i.e., aggressive competitors, this reduces to $I \approx(1-k F) /(1-k S) \approx 1-k(F-S)$, i.e., to the difference between the first and second strikes. $F$ is generally larger than $S$, so the index is less than unity in proportion to the difference between second and first strikes. For $\mathrm{L}$ small, conservative competitors, Eq. (8) reduces to $\mathrm{kS} / \mathrm{L} / \mathrm{kF} / \mathrm{L}=\mathrm{S} / \mathrm{F}$, an index proportional to the ratio of $S$ to $F$, which for $S$ and $F$ large, gives I approaching unity.

Results. The report studies the sensitivity to all of the parameters in Eqs. (1) - (4). The optimal allocation of weapons favors missiles large numbers of weapons per missiles; value at one to two weapons per missile, where the first and second strike costs approach one another and the stability index approaches unity. For fixed numbers of weapons per missile, allocations vary less as the number of missiles is reduced, but the allocation for triplets is only about a third of that for singlets. By $\sim 100$ missiles, the stability indices for a given number of weapons on singlet and triplet missile configurations are comparable-are the number of weapons each would deliver on value targets-which is counterintuitive. Although these results are derived for vulnerable forces, they are generally applicable because of the cancellation of survivable forces from stability indices discussed below.

\section{Probability of Survival from Multiple Weapon Attacks (LA-UR-97-664,} Feb 1997) shows that a missile's survival probability can be approximated with sufficient accuracy by an analytic function of the average number of weapons per missile $\mathrm{W}$ as

$$
\mathrm{Q} \approx \mathrm{q}^{\mathrm{r}} \text {, where } \mathrm{r}=\mathrm{fW} / \mathrm{M} \text {, }
$$

This exponential approximation is $\sim 5-10 \%$ accurate for kill probabilities $\mathrm{p} \sim 0.6, \sim 10-20 \%$ accurate for kill probabilities $\mathrm{p} \sim 0.8$ and $\mathrm{r} \ll 1$, and is well suited to analytic optimization for the $p \sim 0.6, r>1$ conditions of greatest interest below.

6. Stability of Symmetric Missile Forces (LA-UR-97-930, Mar 1997) studies the MIRV to singlet transition for mixes of vulnerable and survivable missiles that represent current configurations. The study uses numerical attack optimization, which is appropriate at current force levels. The optimal allocation of weapons between missiles and value is used in evaluating the stability impact of reducing the number of weapons per missile at large numbers of missiles. For mixes with $50 \%$ survivable missiles, the optimal allocation of weapons to missiles is about $30 \%$, which is about half that for the vulnerable missiles treated in the previous note. For optimized attacks, a reduction in the number of weapons per missiles from 10 to 3 decreases stability slightly; smaller MIRV ratios increase the index to about unity. 
Stability increases as the number of weapons per missile or their kill probability is reduced, relatively insensitive to relative preferences for inflicting damage to the other and reducing damage to self for symmetric forces and objectives. The optimal allocation of weapons between missiles and value is significant in predicting the impact on stability of weapon reductions at large forces, less of an issue in missile reductions at fixed numbers of weapons per missile. At 100 missiles, stability indices for singlet and triplet configurations with survivable forces are comparable, and on the order of those for vulnerable missile forces.

As the total number of missiles is reduced while maintaining $50 \%$ survivability, the optimal allocation for triplet missiles drops, while the optimal singlet allocation remains about constant. In a reduction from 1,000 to 100 missiles on each side, the singlet missile stability index remains near unity, while the triplet index lies about 5-10\% below it. At 100 missiles, the stability indices for $50 \%$ survivable singlet and triplet configurations are comparable and about equal to the stability indices for 1 CO\% vulnerable singlet and triplet configurations, If the goal is low, comparable numbers of missiles and weapons, the distinction between singlets and multiplet configurations and vulnerable and survivable basing does not appear fundamental.

7. Stability of Unsymmetric Forces (LA-UR-97-1133, Mar 1997) derives the equations for exchanges between unsymmetric missile forces and extends the discussion of crisis stability to unsymmetric mixtures of vulnerable and survivable missile forces with varying numbers of warheads. The equations for unsymmetric forces can be derived most simply with a "prime" notation in which the symbols of one side remain as defined above and those of the other side are denoted by primes. This both simplifies the equations and renders them nationality neutral. the exchange equations are more complex. Thus, prime has $\mathrm{M}^{\prime}$ vulnerable missiles with $\mathrm{m}^{\prime}$ weapons each and $\mathrm{N}^{\prime}$ invulnerable missiles with $\mathrm{n}^{\prime}$ weapons each, so the average numbers of weapons delivered on each prime vulnerable missile is

$$
\mathrm{r}=\mathrm{f}(\mathrm{mM}+\mathrm{nN}) / \mathrm{M}^{\prime}
$$

which gives a survival probability of $Q^{\prime} \approx m^{\prime} M^{\prime} q^{r}$ and a prime second strike of

$$
S^{\prime}=m^{\prime} M^{\prime} Q^{\prime}+n^{\prime} N^{\prime} \approx m^{\prime} M^{\prime} q^{r}+n^{\prime} N^{\prime},
$$

on unprime's value targets. The equations for prime's first strike can be obtained either by repeating the above logic from prime's side or by "conjugating" Eqs. (1)-(4), i.e., by replacing primed symbols by unprimed symbols and vice versa. Thus, prime's first strike is

$$
F^{\prime}=\left(1-f^{\prime}\right)\left(m^{\prime} M^{\prime}+n^{\prime} N^{\prime}\right),
$$

and the average number of weapons per unprime vulnerable missile is

$$
r^{\prime}=f^{\prime}\left(m^{\prime} M^{\prime}+n^{\prime} N^{\prime}\right) / M,
$$

which for $r$ large gives $Q^{\prime} \approx q^{\prime} r^{\prime}$, where $p^{\prime}$ is a prime missile's single shot probability of kill on unprime's vulnerable missiles. Unprime's second strike from conjugating Eq. (11) is 


$$
\mathrm{S} \approx \mathrm{mMq}^{\prime} \mathrm{T}^{\prime}+\mathrm{nN}
$$

which is delivered on prime's value targets, as before. These equations are suited for studying force asymmetries of any order. If unprime strikes first on primes vulnerable missiles and $1 / \mathrm{k}^{\prime}$ value targets, that provokes a second strike $S^{\prime}$ from prime, so that the cost to unprime is

$$
C_{1}=\left(1-e^{-k S^{\prime}}+L e^{-k} \mathrm{~F}^{\prime}\right) /(1+L),
$$

where unprime's preference $L$ is used in the combination of costs, as it is he who must make the decision of strike first. If unprime waits and strikes second, his cost is

$$
\mathrm{C}_{2}=\left(1-\mathrm{e}^{-k F^{\prime}}+\mathrm{Le}^{-\mathrm{k}^{\prime} \mathrm{S}}\right) /(1+\mathrm{L}) \text {, }
$$

where unprime's preference is still used in the combination, as it is he who must make the assessment of this cost. The costs for prime can be obtained by conjugation

$$
\begin{aligned}
& C_{1}{ }^{\prime}=\left(1-e^{-k^{\prime} S}+L^{\prime} e^{-k F^{\prime}}\right) /\left(1+L^{\prime}\right), \\
& C_{2}{ }^{\prime}=\left(1-e^{-k^{\prime} F}+L^{\prime} e^{-k S^{\prime}}\right) /\left(1+L^{\prime}\right),
\end{aligned}
$$

The overall stability index becomes the product of each sides' index or

$$
I=\left(C_{1} / C_{2}\right)\left(C_{1}^{\prime} / C_{2}^{\prime}\right) \text {. }
$$

Even if the forces and strikes are symmetric so that $F=F^{\prime}$ and $S=S^{\prime}$, the stability index is not unity unless $\mathrm{L}=\mathrm{L}$ ' as well, i.e., unless the two sides have equal preferences for damage and damage limiting.

8. Stability of Missile Forces and Defenses (LA-UR-97-1223, April 1997) analyzes the impact of defenses on missile mixes with numerical optimization, showing the expected decrease in stability at intermediate levels and recovery for dominant defenses. The analysis assumes that the offensive forces of each side are comparable in size and capability. For equal MIRVing of vulnerable and survivable forces, the exchange equations are a simple extension of those derived previously for mixes of vulnerable and survivable forces. For a total of $\mathrm{D}$ random subtractive defenses that negate the missile before the deployment of warheads, the first strike on value targets is reduced to

$$
F=(1-f)(m M+n N)[1-D /(M+N)],
$$

where the number of weapons is $\mathrm{W}=\mathrm{mM}+\mathrm{nN}$. The effect of such defenses is to reduce the number of both survivable and nonsurvivable offensive missiles by a fraction $\approx 1-D /(M+N)$. If there were $D=M+N$ defenses, the fraction would be zero, and neither sides' missiles would penetrate the defenses. The average number of weapons delivered on each vulnerable missile is

$$
\mathrm{r}=\mathrm{f}(\mathrm{mM}+\mathrm{nN})[1-\mathrm{D} /(\mathrm{M}+\mathrm{N})] / \mathrm{M} \text {. }
$$

For $r$ large, the average probability of survival is approximately $Q \approx q^{r}$, where $p=1-q$ is the attacking missile's single shot probability of kill. The second strike is

$$
\mathrm{S}=(\mathrm{mMQ}+\mathrm{nN})[1-\mathrm{D} /(\mathrm{MQ}+\mathrm{N})] \text {, }
$$


all of which is delivered on value targets. Defenses again subtract proportionally from the number of missiles launched in the second strike. While it is possible to solve these equations for arbitrary parameters, a simple, relevant choice is $m=n$, i.e., equal degrees of deMIRVing on survivable and vulnerable missiles, which reduces Eq. (10) to

$$
F=(1-f) m(M+N)[1-D /(M+N)]=(1-f) m(M+N-D),
$$

so that the first strike is equal to the number of escaping missiles times the number of weapons per missile $\mathrm{m}$ times the fraction directed towards value. For this choice Eq. (21) reduces to

$$
\mathbf{r}=\mathrm{fm}(\mathrm{M}+\mathrm{N}-\mathrm{D}) / \mathrm{M} \text {, }
$$

and Eq. (22) to

$$
\mathrm{S}=\mathrm{m}(\mathrm{MQ}+\mathrm{N}-\mathrm{D}) \text {, }
$$

which is the number of surviving missiles less the number of defenses times $\mathrm{m}$ weapons per missile. For equal MIRVing of vulnerable and survivable forces, the exchange equations are a simple extension of those derived previously for mixes of vulnerable and survivable forces. For fixed missile numbers, increasing defenses first decreases strike sizes, costs, and stability due to the preferential erosion of second strikes by the defenses. For fixed defenses, decreasing the number of missiles decreases stability to the point where first strikes fall to zero. Making the defenses larger could effect that transition at a larger number of missiles. A judicious increase in defenses and decrease in offenses could effect that transition with minimum loss of stability.

9. Sensitivity to Attack Preferences (LA-UR-97-1224, April 1997) studies the variation of strikes and indices for symmetric missile mixes with numerical optimization of attacks. It uses the exchange equations derived earlier for mixes of missiles without defenses, optimizing strikes for every value of the "relative attack preference" parameter. The report discusses the sensitivity of stability indices to relative preference between inflicting damage on others and preventing damage to oneself, which was identified as a key uncertainty in earlier analytic models. Figure 3 of the report shows that stability falls for large attack preferences. That is a major concern of Russian analysts, as discussed in conjunction with the first three reports on their simplified model, which also exhibits this effect.

The calculations show that conservative estimates of the other side's preference could reduce stability indices by about $50 \%$, which is as large an effect as produced by other factors studied. The reduction is caused by the optimization's shifting weapons from missile to value targets in order to score additional value. That increases first strikes more than second, which reduces stability, although both first and second strike costs fall significantly. To oversimplify, very aggressive opponents largely ignore the opportunity to limit damage and destroy as much value as possible.

10. Unacceptable Damage as an Analysis Tool (LA-UR-97-1353, April 1997) shows that a criteria of unacceptable damage removes all freedom in attack optimization and 
leads to attacks purely on value for small missile mixes. Equations (10) - (19) constitute a closed model that can be solved to predict the variation of $\mathrm{C}_{1}, \mathrm{C}_{2}$, and I with model parameters. If the goal is to inflict unacceptable damage $F_{u}$ in the first strike, $F$ is fixed at that level, and

$$
\begin{aligned}
& \mathrm{f}=1-\mathrm{F}_{\mathrm{u}} / \mathrm{W}, \\
& \mathrm{r}=\mathrm{fW} / \mathrm{M}=\left(1-\mathrm{F}_{\mathrm{u}} / \mathrm{W}\right) \mathrm{W} / \mathrm{M}=\left(\mathrm{W}-\mathrm{F}_{\mathrm{u}}\right) / \mathrm{M} .
\end{aligned}
$$

As the desired damage $F_{u}$ increases, the allocation to missiles $f$ and the weapons per missile $r$ fall linearly. The first strike is constant at the desired $\mathrm{F}_{\mathrm{u}}$; the second strike is much larger for ratios larger than doublets. As the number of weapons per missile decreases, $f$ is roughly constant at large numbers of weapons per missiles, but decreases sharply below two weapons per missile, reaching $\approx 0$ when the number of attacking weapons equals $F_{u}$. The first strike cost is much larger than the second strike cost for many weapons per missiles, so the first striker would be deterred from initiating such a disadvantageous engagement.

The criteria that one be able to inflict unacceptable second strike damage, $S_{\mathrm{u}}$, implies

$$
\begin{aligned}
& \mathrm{S}_{\mathrm{u}}=\left(\mathrm{mMq}^{\mathrm{r}}+\mathrm{nN}\right), \\
& \mathrm{q}^{\mathrm{r}}=\left(\mathrm{S}_{\mathrm{u}}-\mathrm{nN}\right) / \mathrm{mM}, \\
& \mathrm{r}=\ln \left[\left(\mathrm{S}_{\mathrm{u}}-\mathrm{nN}\right) / \mathrm{mM}\right] / \ln \mathrm{q},
\end{aligned}
$$

where $r=f(m M+n N) / M$, which places a limit on the opponent's maximum allowable $f$, which determines whether or not the second striker can meet $\mathrm{S}_{\mathrm{u}}$. Figure 3 of the report shows $\mathrm{F}$ and $\mathrm{S}$ as functions of $f$. $F$ falls as missiles are reallocated to value. $S$ falls for small $f$ but asymptotes to $\mathrm{nN}$, the number of survivable weapons by $\mathrm{f} \sim 0.5$. Thus, unacceptable second strike damage can only be guaranteed by adequate survivable forces, although it can be achieved provisionally if the attacker allocates a large fraction of his weapons to value, which is not always optimal.

Overall, unacceptable damage has no slack and does not permit optimization of attacks. In first strikes, it is inferior to the cost minimization for large values of forces and marginal for small forces. In second strikes, it can only be guaranteed through adequate survivable forces.

\section{Optimal Time Dependent Deployment of Missile Forces (LA-UR-97-} 1379, April 1997) is a preliminary investigation of optimum arms control trajectories beyond START using numerical inter-temporal optimization of first strike costs for symmetric forces. It extends the previous static force exchange and stability calculations to the optimal deployment of forces over time by adding equations for growth and decay of missiles and minimizing the integral of first strike cost over the time interval of interest, while varying the allocations of first strikes to missiles and missile purchase rates in each period. Equations (10) - (19) can be solved to evaluate $\mathrm{C}_{1}, \mathrm{C}_{2}$, and $\mathrm{I}$ at any time $\mathrm{t}$ given $\mathrm{M}, \mathrm{m}, \mathrm{N}, \mathrm{n}, \mathrm{p}$, and $\mathrm{L}$. They are extended by the inclusion of an equation for the rate of change of missiles, assuming $M=N$ and $m=n$, 


$$
\begin{aligned}
& \mathrm{dM} / \mathrm{dt}=\mathrm{P}-\mathrm{M} / \mathrm{T}, \\
& \mathrm{dC} / \mathrm{dt}=\mathrm{cP},
\end{aligned}
$$

where $P(t)$ is the number of missiles purchased in year $t, T$ is the average lifetime of a missile, and $c$ is the average missile + weapon + operational lifetime cost of a missile. The extension to time dependent forces is accomplished by minimizing the integral of $\mathrm{C}_{1}$ over the time interval of interest by varying $P$ and $f$, subject to appropriate constraints on total cost $C$.

For $\mathrm{L}<1, \mathrm{M}$ decays exponentially, as missiles assessed to. be ineffective by nonaggressive opponents are allowed to decay. For $\mathrm{L}=1$, i.e., opponents indifferent between producing and avoiding damage, $M$ reaches a steady state at a level slightly lower than the initial one. For $\mathrm{L}>1, \mathrm{M}$ increases quickly to higher levels where it saturates due to nonlinear effects. For $\mathrm{L}=1.25-1.5$, $\mathrm{I}$ increases to about unity in a few years. For $\mathrm{L}=1, \mathrm{I}$ decreases. For $\mathrm{L}=$ 0.75 , I initially falls, but then increases to $\sim 1$. Either a decaying number of missiles and small $L$ or a growing one and large L lead toward stability: While similar from a stability perspective, these two cases involve very different numbers of delivered weapons in a conflict. The intermediate $L=1$, which represents indifference to damage to other and self, leads to a stable number at a slightly reduced stability index with an appreciable number of weapons.

\section{Attack Optimization at Moderate Force Levels (LA-UR-97-1523, April} 1997) derives the analytic optimization of attack allocation for symmetric mixes of missiles with and without defenses and shows that the analytic results are accurate for $\sim 1,000$ weapons. It derives optimal offensive missile allocations between missiles and value forces for moderate offensive and defensive forces. For vulnerable missiles it is possible to analytically derive the value of the allocation that minimizes the first strike cost for any given attack preference, number of weapons per missile, and kill probability. For moderate forces, these vary strongly with attack preference for few weapons per missile and fall more slowly for larger numbers. The attacker wishes to minimize $\mathrm{C}_{1}$, which for moderate forces reduces to

$$
\mathrm{C}_{1}=[\mathrm{L}+\mathrm{k}(\mathrm{S}-\mathrm{LF})] /(1+\mathrm{L})=\left\{\mathrm{L}+\mathrm{k}\left[\mathrm{mMq}^{\mathrm{fm}}-\mathrm{L}(1-\mathrm{f}) \mathrm{mM}\right\} /(1+\mathrm{L}),\right.
$$

whose minimum can be found by differentiation with respect to $\mathrm{f}$ and setting the result to zero as

$$
\mathrm{f}_{\mathrm{O}}=\ln (-\mathrm{L} / \mathrm{m} \ln \mathrm{q}) /(\mathrm{m} \ln \mathrm{q})
$$

which depends inversely on $\mathrm{m}$, but only logarithmically on $\mathrm{L}$ and $\mathrm{q}$. The optimal $f_{\mathrm{a}}$ can be substituted into Eq. (33) to determine the value of $\mathrm{C}_{1}$ corresponding to that $\mathrm{m}$, which is

$$
\mathrm{C}_{1}=\left\{\mathrm{L}+\mathrm{kmM}\left[\mathrm{q}^{\mathrm{fm}}-\mathrm{L}(1-\mathrm{f})\right]\right\} /(1+\mathrm{L}),
$$

in which $\mathrm{L} /(1+\mathrm{L})$ is only a function of $\mathrm{L}$ and the second term is a product of $\mathrm{kmM}$ and a function of $m, L$, and $q$. For a given $L$, the derivative of $C_{1}$ with respect to $M$ is

$$
\partial \mathrm{C}_{1} / \partial(\mathrm{kM})=\mathrm{m}\left[\mathrm{q}^{\mathrm{fm}}-\mathrm{L}(1-\mathrm{f})\right] /(1+\mathrm{L}) .
$$


For positive values of the derivative, the first striker should minimize first strike costs by reducing the number of missiles, as $(\mathrm{dM}) \partial \mathrm{C}_{1} / \partial \mathrm{M}$ would then be negative, which would reduce $\mathrm{C}_{1}$. For negative values of the derivative, he should minimize costs by increasing the number of his missiles and warheads, as the product $(\Delta \mathrm{M}) \partial \mathrm{C}_{1} / \partial \mathrm{M}$ would be negative, which would again reduce $C_{1}$. Thus, the combination of parameters for which the derivative is zero defines the boundary between temporal growth or decay of offensive forces seen in the previous study. The transition occurs at roughly the current weapons per missile and attack preference.

Survivable missiles make the derivative positive for all values of weapons per missile for small attack preferences, although they are still negative for large values. The transition occurs at the attack preference where the first striker gives roughly equal priority to inflicting damage on the other and preventing damage to himself. To apply these results it is necessary to have an estimate of the opponent's preference, which cannot be known with certainty. Figure 7 shows that defenses make the derivative smaller as defenses increase. There is a transitional region in which the derivative-and hence the incentive for missile growth-is small for all defenses, but occurs at a smaller attack preference than assumed in the absence of defenses.

\section{Optimization of Stability Index Versus First Strike Cost (LA-UR-97-} 1888, May 1997) compares indices from minimizing first strike costs with those from maximizing overall stability. To maximize stability, the attacker would normally determine the $f$ that maximizes the stability index I analytically; however, here differentiation generally produces the minimum of $I$. The maximum is produced by setting $f=1$, which produces

$$
\mathrm{I}_{\max } \approx \mathrm{kmMq}^{\mathrm{m}} \text {, }
$$

for $L / k \gg F, S$. The index from maximizing $I$ is $\sim 1$; the index from minimizing $C_{1}$ falls. The difference between them-and the penalty in I for minimizing $\mathrm{C}_{1}$-grows to $\sim 15 \%$ for intermediate $\mathrm{L}$. If each side could trust the other to maximize stability by minimizing damage to value, I could be increased by about $15 \%$. However, that also would increase the cost to the first striker by about $15 \%$. It is unclear why-having decided to strike-the first striker would do so in a way that would reduce the effectiveness of his attack and increase damage to himself.

14. Effect of Unsymmetric Missile Force Reductions (LA-UR-97-2194, June 1997) uses the above models to study the impact of unilateral U.S. reductions of weapons per missile. Forces fall in equal decrements from START I to II levels. The decrementing side's average optimal attack allocation to missiles is about 0.5 ; the static side's about 0.25 . The static side's first and second strikes are roughly constant; the decrementing side's first and second strikes drop in proportion to his decreasing fractionation. By the end of the transition, the static side could inflict about a factor of 2 more damage by striking first. The decrementing side could achieve parity in damage only by striking first. 
The static side's second strike costs fall to his first strike costs, and the decrementing side's first strike costs rise toward his second strike costs, but do not reach them by START II. The static side reaches unit stability; the decrementing side reaches $\sim 0.8$. Overall, decreasing vulnerable forces increases the cost of striking first, and decreasing survivable forces reduces second strikes. The principal result is that a large, unilateral U.S. reduction would increase overall stability at the expense of U.S. first and second strikes.

15. Attack Optimization for Unequal Moderate Forces (LA-UR-97-2195, June 1997) extends the analytic attack optimization of Eqs. (33) - (36) to unequal forces using the cost equations derived in Eqs. (15) - (18) to study the variation of strikes and indices with the number of triplet missile mixes, finding instability at both large and small numbers of missiles. Unsymmetric forces simply generalize Eq. (34) to

$$
f_{0}=\left(M^{\prime} / W \operatorname{lnq}\right) \ln \left(-L^{\prime} / k m^{\prime} \operatorname{lnq}\right) \text {, }
$$

so that unprime's optimal allocations scale directly on the opponent's vulnerable missiles, inversely on his own total weapons, and logarithmically on damage preference, kill probability, and relative target sets. Prime's allocation can be obtained by conjugation. It scales in a similar manner on the unprime's parameters. First and second strikes increase for the side with greater forces and decrease for the side with less. Conversely, first and second strikes decrease for the side with greater forces and increase for the side with fewer. If one side's forces are much larger, his costs drop to levels where he is insensitive to whether he strikes first or second. Stability indices exhibit a minimum where the two sides have roughly equal forces.

16. Sensitivity of Stability Indices to Force Uncertainties (LA-UR-97-2689, July 1997) studies the variation of strikes, costs, and indices with hidden weapons or missiles during proportional missile reductions. Additional vulnerable weapons in the inventory of the side with the greater fraction of vulnerable forces, i.e., Russia, would produce a shift to value in Russian second strikes and a larger shift to missiles in U.S. first strikes, which increases second strike costs more than first, which is destabilizing, but additional Russian weapons would increase stability indices by compensating for their inferior initial position.

Additional survivable weapons produce a shift to value in Russian second strikes but no shift in the U.S. first strike, which would use them on value in either case. Additional survivable weapons cause the opponents first strike costs to increase faster than his second strike costs, which reduces the perception of advantage to preemption, increasing stability.

When one side has mostly vulnerable forces, obtaining additional vulnerable weapons appears stabilizing, largely as a compensation for his initial inferiority. For survivable weapons, stabilization is again a compensation for initial inferiority, but is, and provides useful increments to stability indices that are less sensitive to model parameters at intermediate steps in force reductions, where indices would otherwise decline to bothersome levels. 
17. First Strike Stability at Low Weapon Levels (LA-UR-97-2690, July 1997) applies the above unequal force model and optimization to study proportional reductions in vulnerable weapons and missiles from 1,000 to 100. At and below START II force levels, cost and index equations can be linearized, and optimal attack allocations found analytically. Allocations are similar until the end of the transition, when the side with more survivable forces shifts toward damage limiting and the side with fewer shifts towards value. First and second strike costs remain significant and favor the side with fewer vulnerable forces. Proportional reductions reduce stability by giving the side with fewer vulnerable weapons a benefit in preemption. That benefit would be reduced if both sides shifted towards survivable forces during the reduction. Reductions in vulnerable weapons and missiles decrease U.S. and overall stability during transition, although they recover at single weapon missiles.

18. Stability Issues in Reconstitution by Weapon Addition (LA-UR-973149, August 1997) gives an analytic derivation and discussion of the variation of strikes, costs, and indices for missile mixes small enough for exchange and cost equations to be linearized, conditions for which are discussed in the following report. In this limit, the stability index is proportional to the difference between the second and first strikes and can be expressed directly in terms of the number of vulnerable weapons and missiles. Survivable forces cancel out of expressions for stability indices at low forces

$$
\begin{aligned}
(\mathrm{I}-1) \mathrm{L} & \approx \mathrm{k}\left(\mathrm{Q}^{\prime} \mathrm{m}^{\prime} \mathrm{M}^{\prime}+\mathrm{n}^{\prime} \mathrm{N}^{\prime}\right)-\mathrm{Lk}\left(\mathrm{W}-\mathrm{r} \mathrm{M}^{\prime}\right)-\left[\mathrm{k}\left(\mathrm{W}^{\prime}-\mathrm{r}^{\prime} \mathrm{M}\right)-\mathrm{Lk}(\mathrm{QmM}+\mathrm{nN})\right] \\
& =\left(-\mathrm{Lk}^{\prime} \mathrm{m}+\mathrm{kr}+\mathrm{Lk} \mathrm{k}^{\prime} \mathrm{Q}\right) \mathrm{M}+\left(\mathrm{k} \mathrm{Q}^{\prime} \mathrm{m}^{\prime}+\mathrm{Lk} \mathrm{k}^{\prime} \mathrm{r}-\mathrm{km}\right) \mathrm{M}^{\prime},
\end{aligned}
$$

in which survivable forces, $N^{\prime}$ and $N$, appear in the first line but not in the second due to the cancellation between $S^{\prime}$ and $F^{\prime}$ and $S$ and $F$, in the second, which results from all survivable forces being used in both first and second strikes. Since the number of survivable forces in first and second strikes is the same, they do not impact stability.

These results can be used to discuss the impact of the one-sided addition of weapons to initially equal numbers of vulnerable and survivable weapons. The side that adds weapons has more weapons and allocates a greater fraction of them to counter value. Second strikes do not change when reconstitution is achieved by fractionation. The side that does not fractionate has decreasing first and second strike costs. Both sides' stability indices fall, but the fall is smaller for the side that fractionates. Thus, overall stability index falls significantly as the number of weapons per missile increases.

19. Limits on Linearity of Missile Allocation Optimization (LA-UR-973569, August 1997) examines the limits on linearization of the missile exchange cost model used to optimize missile attack allocation, shows that it is accurate for numbers of weapons on missiles below the current $\sim 1,000$ value targets, and concludes that it can be used to bridge the gap between START III levels and the deep reduction regime, where it is asymptotically exact. 
The report also addresses why each sides' optimal first strike is determined by the number of the other side's vulnerable missiles and warheads—rather than his own - and why each side's second strike is independent of his weapon uploading.

For 100 vulnerable and 100 survivable missiles on each side, analytic optimization agrees closely with the numerical solution. By 200 vulnerable and 200 survivable missiles per side, stability indices derived from analytic optimization agrees to those from numerical optimization to within $\sim 10 \%$, but the analytic allocations for the side with more weapons disagree with the analytic allocations by up to $50 \%$. This discrepancy results from the interaction of two effects: the additional weapons and the reallocation of weapons from missiles to value, which they induce.

20. Stability at Low Symmetric force Levels (LA-UR-97-3728, September 1997) studies strikes and stability at force levels well below the number of value targets. As the number of weapons per vulnerable missile decreases, configurations approach stability. For small forces, survivable forces cancel out of stability indices, which reduce to bilinear products of the number of vulnerable missiles and the difference between the normalized second and first strikes. From a stable configuration in which this difference is zero, increasing the number of singlet missiles would not alter stability, but increasing the number of weapons per vulnerable missile would decrease stability. With optimal allocation, for symmetric forces the first strike is

$$
\mathrm{F}=(1-\mathrm{f}) \mathrm{W}=[1-(\mathrm{M} / \mathrm{W} \operatorname{lnq}) \ln (-\mathrm{L} / \mathrm{mlnq})] \mathrm{W}=\mathrm{mM}+\mathrm{nN}-(\mathrm{M} / \operatorname{lnq}) \ln (-\mathrm{L} / \mathrm{mlnq}),
$$

and the second strike is

$$
\mathrm{S}=\mathrm{QmM}+\mathrm{nN}=-\mathrm{ML} / \mathrm{lnq}+\mathrm{nN} \text {. }
$$

The difference between them is

$$
\begin{aligned}
\mathrm{S}-\mathrm{F} & =\mathrm{QmM}+\mathrm{nN}-[\mathrm{mM}+\mathrm{nN}-(\mathrm{M} / \mathrm{lnq}) \ln (-\mathrm{L} / \mathrm{mlnq})] \\
& =\mathrm{mM}[\mathrm{Q}-1+\ln (-\mathrm{L} / \mathrm{mlnq}) / \mathrm{mlnq}],
\end{aligned}
$$

in which the number of survivable weapons cancels out, as the same number is in the first and second strikes. Thus, $\mathrm{S}-\mathrm{F}$, and hence the stability index, depend only on $\mathrm{m}, \mathrm{M}, \mathrm{L}$, and q. Given the form of Eq. (42), it is convenient to define the index

$$
\mathrm{j}=\mathrm{J} / \mathrm{kM}=\mathrm{s}-\mathrm{f}=\mathrm{Qm}-(\mathrm{m}-\mathrm{r}),=
$$

where $s$ and $f$ are the normalized second and first strikes due to vulnerable missiles. $j$ depends bilinearly on $\mathrm{s}-\mathrm{f}$, where $\mathrm{s}$ depends on $\mathrm{L} / \mathrm{lnq}$ and $\mathrm{f}$ on $\mathrm{m}, \mathrm{L} / \mathrm{m}$, and $\mathrm{q}$. Thus, decreasing $\mathrm{p}$ increases stability. Increasing $L$ shifts weapons from counterforce to countervalue, but has little overall impact on stability.

$\mathrm{Q}$ falls as $1 / \mathrm{m}$, and the number of weapons allocated to each vulnerable missile increases as $\mathrm{r} \sim \ln \mathrm{m}$, so the second strike $\mathrm{s}=\mathrm{mQ}=-\mathrm{L} / \mathrm{lnq}$ is constant. But $\mathrm{f}=\mathrm{m}-\mathrm{r} \sim \mathrm{m}$; thus, $\mathrm{j}=\mathrm{s}-\mathrm{f}$ becomes negative, indicating instability. The current configuration involves many missiles and weapons per missile. There are three ways to approach stability: 1 . reduce the number of 
missiles to zero, which produces stability trivially - at the price of increased sensitivity to hidden weapons. 2. reduce $\mathrm{s}-\mathrm{f}$ to zero, which can be accomplished by reducing $\mathrm{m}$ to unity for any M. 3. use inaccurate vulnerable missiles or disperse targets; however, both are sensitive to the value of kill probability, which cannot known with certainty.

The goal of most arms-control discussions is a configuration with a modest number of singlet vulnerable missiles in a mix with many survivable weapon, which is stable. Increasing the number of vulnerable single weapon missiles would not alter the stability index since $\Delta \mathrm{J}=$ $\Delta \mathrm{M}(\mathrm{s}-\mathrm{f})=\Delta \mathrm{M}(0)=0$. However, for any $\mathrm{M}$, increasing $\mathrm{m}$ decreases stability. By $\mathrm{m}=2$, the decrease is $\sim-0.5 /$ missile. Thus, 200 vulnerable missiles would decrease the index by $\approx \mathrm{kM} \Delta \mathrm{j} \approx$ $0.001 \times 200 \times(-0.5) \approx 0.1$ for only an additional $\sim 200$ warheads. As the number of weapons per vulnerable missile decreases, the configuration becomes stable for any number of missiles. Increasing the number of vulnerable single-weapon missiles would not alter stability, but increasing the number of weapons per vulnerable missile would decrease stability.

\section{Stability of Nuclear and General Purpose Forces (LA-UR-97-4618,} November 1997) uses the model for nuclear missile exchanges to describe the interaction between a force with nuclear weapons and another with conventional superiority. The two are rationalized in terms of nuclear equivalents, and extended conflicts are modeled in terms of effective first and second strikes. Nuclear weapon allocations are proportional to conventional forces, but saturate with all weapons on military value to limit damage. Conventional allocations to nuclear forces fall as conventional forces increase, reflecting a shift from damage limiting to inflicting as much damage as possible with the forces that survive nuclear attack.

The kill probability of nuclear weapons degrades against dispersed forces. As they become ineffective against dispersed forces, that they are reallocated to value targets. Since forces might be dispersed after the commencement of hostilities, this implies urgency in the use of nuclear weapons before they lose effectiveness. The stability of roughly equal-force configuration is neutral for small nuclear kill probabilities, but it results from a cancellation between stability seen by the nuclear forces with that seen by conventional forces. Greater conventional force effectiveness decreases the stability index.

Decreasing the effectiveness of conventional forces against weapons increases stability; e.g., if the conventional forces have few precision or deep-strike weapons, or if the nuclear weapons are well dispersed. However, stability falls as the nuclear kill probability increases and as nuclear forces make a greater contribution, which means introducing nuclear forces into a conventional engagement, or making them more effective, is destabilizing because they suppress the conventional second strike. Given the great degradation nuclear forces could inflict on conventional forces before they were dispersed, the conventional force commander has a 
great incentive to eliminate them, which would start the war, or disperse, which would reduce the effectiveness of nuclear forces, giving the nuclear forces an incentive to strike first.

These results roughly model concerns in current theaters. For decades, the US has used the threat of use of nuclear weapons in Europe as an offset to NATO's numerical inferiority in conventional forces, linking their use to US strategic nuclear forces to suppress the stability implications. Russia has recently indicated that qualitative improvements in US forces necessitate a shift to a greater reliance on nuclear weapons to stabilize the theater. The impact of this shift can be understood by interchanging the roles of the two sides above.

The extension of the nuclear crisis stability formalism to nuclear and conventional elements is natural and appears fruitful. It is straightforward to relate conventional maneuver units and capabilities to missile numbers and weapons. The major new elements are the reduced effectiveness of nuclear weapons against dispersed forces and the lower relative effectiveness of conventional weapons against missiles or bases. Both can be treated with the kill probabilities in the strategic missile formalism. For roughly equal-capability forces, nuclear kill probabilities can offset the effects of greater conventional force dispersion and dispersal, but they imply significant reductions in stability unless offset by survivable or out of theater forces.

\section{Stability of Nuclear and Forces and Weapons of Mass Destruction} (LA-UR-97-, December 1997) uses the nuclear crisis stability formalism study exchanges involving chemical and biological weapons of mass destruction (WMD). In theater engagements, where nuclear weapons and troops are imperfectly protected, WMD provide a useful alternative to nuclear weapons. The analysis need only treat in-theater force, as ex-theater forces, which enter as survivable forces, cancel out. Optimal nuclear weapon and WMD allocations are proportional to the opponent's carriers and inversely proportional to one's own weapons. Nuclear weapon kill probaiilities degrade rapidly against dispersed forces, which produces an incentive for the nuclear side to use his weapons while they are still effective. As they lose effectiveness, they are reallocated to value, and have little direct impact on the WMD.

The stability of singlet WMD forces is negative for the small probabilities corresponding to the expected full dispersal, where it is dominated by the WMD. For triplet WMD carriers, stability is determined about equally by nuclear and WMD at large kill probabilities. It is determined by WMD at small kill probabilities. That an opponent might use a different stability calculus can be modeled through model parameters that represent damage preference. An opponent with WMD and a strong preference for damage would shift his allocation from missiles to value to increase his first strikes on value by a like amount, which would decreases stability at large kill probabilities, where deterrence is generally thought to be effective.

Summary and conclusions. This note reviews a collection of papers that discuss the stability of combinations of nuclear, conventional, and WMD forces. The framework used is an 
extension of that in previous U.S. analysis, which incorporates the metric used to allocate attacks identified in Russian publications. The analysis used here is for two-sided interactions, for which the model can be reduced to 7 equations for the first and second strikes, costs, and stability indices for each side, which involves 6 main parameters. If 5 values of each parameter are used to crudely characterize various configurations, the number of possibilities, or rough dimensionality of the analysis, is $\sim 2$ sides x 6 parameter ${ }^{\wedge} 5$ values/parameter $\sim\left(5^{6}\right)^{2} \sim 10^{8}$, which is much large to search blindly.

In reducing this dimensionality and seeking guidance for practical searches, four principles have been of significant value: 1 . minimization of first strike costs to optimize first strikes, costs, and exchanges, 2. simplification of exchanges into broad classes, 3 . treating time dependence by adding inventory models for weapons purchased and optimizing first strike costs over the interval of interest, and 4. linearization of costs indices at low levels, which permits exact optimization of attacks, temonstration of the role of survivable forces drop, and bilinear stability analyses that separates the roles of missiles and weapons in stability and reconstitution.

1. Minimizing first strike costs to optimize first strikes and exchanges is fundamental in reducing dimensionality and introducing structure into the analysis. Other metrics provide valuable contrast, but in the end, minimization of first strike cost as described in the three papers on the Russian model, provides a logical and robust means of optimizing engagements. It also provides a simple framework for evaluating other metrics such as maximizing overall stability or inflicting unacceptable damage, clearly showing that in optimized exchanges, the first striker does what he will, the other side what he must.

Analytic optimizations possible at low force levels exhibit the dependence of optimal allocations on the ratio of an opponent's vulnerable missiles to one's own total weapons as well as its logarithmic dependence on other attack and preference parameters. The "relative attack preference" parameter shows the sensitivity of stability indices to relative preference between inflicting damage on others and preventing damage to oneself, which is identified as a key uncertainty in these models. Stability falls for large attack preferences, which is a major concern of Russian analysts. Overly conservative estimates of the other's damage preference could reduce stability indices by $\sim 50 \%$, which is as large an effect as produced by any other factor studied. Moreover, the reduction is caused by the optimization shifting weapons from missiles to value, which increases first strikes more than second and reduces stability. Aggressive opponents largely ignore the opportunity to limit damage and destroy as much value as possible.

The model analysis permits the derivation of the derivative of first strike cost with respect to the number of missiles and weapons, which defines the boundary between growth or decay of offensive forces. It also shows how survivable missiles and defenses can improve stability. Unfortunately, the apf'ication of these results requires a good estimate of one's 
opponent's preference, which cannot be known with certainty. These results extend naturally to unequal forces, where if one side has much larger forces than the other, his costs drop to levels low enough that he is relatively insensitive to whether he strikes first or second, so that stability indices exhibit a minimum where the two sides have roughly equal forces.

2. Exchanges and indices simplify into broad classes-particularly as the number of weapons and missiles are reduced-which simplifies the dependence of results on target set size and composition and makes it possible to see the essential dependence on a few key parameters. In most cases they can be collapsed to simple dimensionless ratios. These parameters are generally a good guide to trends even when forces are too large for them to be derived precisely. The optimal allocation of weapons shifts from vulnerable missiles at large numbers of weapons per missiles to value at a few weapons per missile. Allocations vary less as the number of missiles is reduced; roughly inversely with the number of weapons per missile. At low levels, the stability indices singlet and triplet missile forces are comparable, as are the number of weapons each would deliver on value. Thus, if the goal is low, comparable numbers of missiles and weapons, the distinction between singlets and multiplet configurations and vulnerable and survivable basing does not appear fundamental.

For equal, fixed forces of multiple weapon vulnerable and survivable missiles, introducing defenses increases allocations to counterforce, which decreases strike sizes, costs, and stability due to their preferential erosion of second strikes. reduction in stability can be quite large at intermediate values of defenses, although for large defenses the stability index recovers to unity for both sides. Varying metrics have impacts similar to defenses. Unacceptable Damage as an Analysis places a limit on the opponent's maximum allocation, as his choice determines whether or not the second striker can meet his damage objective. Unacceptable Damage could be achieved provisionally if the attacker allocated his weapons to value, but it could only be guaranteed by adequate survivable forces. Similarly, optimizing the stability index rather than minimizing the first strike costs sounds laudable, but there is a significant penalty to first striker for doing so, so it is unclear why the first striker-having decided to strike-would do reduce the effectiveness of his attack and hence increase damage to himself.

Missile and weapon reductions are another appropriate application. It is readily shown that in a unilateral reduction from START II to III levels by the U. S. Russian first and second strikes would be about constant and the U.S.'s first and second strikes would fall roughly in proportion to the decreasing number of weapons. At the end of the transition, the U.S. could achieve parity in damage only by striking first. Overall, a large unilateral U.S. reduction could increase overall stability at the expense of U.S. first and second strikes. An interesting corollary is that first and second strike magnitudes decrease for the side with greater forces and increase for the side with fewer, so if one side has much larger forces than the other, his costs drop to 
levels low enough that he is relatively insensitive to whether he strikes first or second. Thus, stability indices exhibit a minimum where the two sides have roughly equal forces.

These results are also sensitive to hidden weapons or missiles. Logically, hidden vulnerable Russian weapons would increase stability indices by compensating for Russia's inferior initial stability - which is due to Russia having a much larger fraction of its weapons on vulnerable rather than survivable carriers. For survivable weapons, stabilization again compensates for this initial inferiority, but could provide useful increments at intermediate steps to stability indices, which could otherwise decline significantly.

3. Time dependency and latency-which are two of the key new variables introduced by this analysis-represent the third guide, which can be treated by an extension of the above exchange models derived by the addition of inventory models for the weapons and missiles purchased and the inter-temporal optimization of the resulting first strike stability in each period. Missile trajectories and stability results break into three regions: 1 . for $\mathrm{L}<1, \mathrm{M}$ decays exponentially, 2 . for $L=1, M$ reaches a steady state at a level near the initial one, 3 . for $\mathrm{L}>1, \mathrm{M}$ increases sharply. Both the decaying number for small $\mathrm{L}$ and the growing one for large $L$ lead toward stability, although they involve very different numbers of delivered weapons in a conflict. The intermediate $L=1$ leads to a stable number at a slightly reduced stability index with an appreciable number of weapons.

These results provide a vehicle for predicting the time scale for unstable latent interaction as well as a vehicle for inferring the damage preference of one's opponent from his responses to changes in deployment. The latter suggests a rough connection to game theoretical treatments of the connection between first strike and crisis stability, in which such moves are used to infer the other's intent and resolve, i.e., damage preference. reconstitution

4. Linearity of costs and indices at low levels provides the fourth guide, as it permits exact optimization of attacks, analytic demonstration the role of survivable forces, and a derivation of bilinear stability analyses that clearly delineate the respective roles of missiles and weapons in stability and reconstitution.

Proportional reductions in vulnerable weapons and missiles reduce stability by giving the side with fewer vulnerable weapons a significant benefit in preemption. That benefit would be reduced if both sides shifted towards survivable forces during the reduction. Reductions in vulnerable weapons and missiles would decrease U.S. and overall stability slightly during transition, although stability indices would recover at single weapon missiles.

For small forces, the stability index is determined by the number of vulnerable weapons and missiles, as survivable forces cancel out of the analysis. Indices reduce to bilinear products of the number of vulnerable missiles and the difference between the normalized second and first strikes by vulnerable weapons $M(s-f)$. Thus, there are three ways to produce stability. The first 
is to reduce the number of missiles to zero, which produces stability trivially at the price of sensitivity to hidden weapons. The second is to reduce $\mathrm{s}-\mathrm{f}$ to zero, which can be accomplished by reducing $\mathrm{m}$ to unity at any $\mathrm{M}$. The third is to use inaccurate missiles or hidden launchers, which are sensitive to the kill probability and concealment, neither of which is known. From $\mathrm{s}-\mathrm{f}$ $=0$, increasing the number of vulnerable single-weapon missiles would not alter stability, but increasing the number of weapons per vulnerable missile would decrease stability. Thus, reconstitution of weapons is more of a concern than of missiles at low force levels.

These models can be applied directly to other types of weapons. For general purpose forces, the main issue is properly relating nuclear and conventional force capabilities. WMD are quite adequate for theater operations against exposed forces. For both, the main issues are the vulnerability and effectiveness of in-theater nuclear forces and the degradation of the kill probability of nuclear weapons against dispersed conventional and WMD forces. Even when their kill probability is high against undispersed forces, nuclear weapons degrade stability strongly because of their great capability coupled with their vulnerability. As their kill probability falls, nuclear weapons are reallocated to value, where they no longer directly impact the non-nuclear forces. Since conventional and WMD forces would be dispersed after the commencement of hostilities, if not before, this loss of effectiveness implies some urgency in the use of nuclear weapons before they lose effectiveness.

These results for nuclear versus conventional forces roughly model stability concerns in current theaters. For decades, the US has used the threat of use of nuclear weapons in Europe as an offset to NATO's numerical inferiority. In the aftermath of the qualitative superiority demonstrated by U.S. forces in the Gulf War, Russia has indicated an interest in using nuclear weapons to compensate for its perceived inferiority. The analysis permits one to view this issue from either side. These models can also be used to explore the possibility that an opponent might use a different calculus to arrive at a different stability assessment, which can be modeled through the parameters in the model that represent relative damage preference. An irrational decision maker can be modeled as a very aggressive opponent, whose shift of weapons from forces to value could significantly decrease stability in the region where it is generally thought deterrence should be effective.

These four guides have made it possible to search efficiently models for a range of missile forces and metrics to produce a fairly simple assessment of the stability properties of this class of models. Using the U.S. exchange models and costs and Russian attack optimization made it possible to connect the two efficiently and provided the first strike cost derivative information that defines the boundary between growth or decay of offensive forces. The results require an estimate of the opponent's damage preference, but they provide a simple framework for assessing sensitivity to it and incorporating information on it. 
Model calculations with various missile and weapon mixes provide make it possible to see essential interrelationships directly. In most cases they can be collapsed to simple dimensionless ratios. The basis for and effect of changing allocations then becomes clear, as do the reasons for the intermediate dip in stability as defenses are introduced, the loss of flexibility under unacceptable damage, and the inaccessibility of maximizing stability as a targeting metric. The problems and payoffs of proportional or unilateral arms reductions provide a fairly simple picture, as do their sensitivity to hidden or added weapons.

Time dependence is an important and interesting subject that is treated well by this analysis, although it has not been explored fully. Calculations have explored the sensitivity of missile buildups or decays to a few simple parameters-primarily damage preferences. The results are plausible and in accord with Russian concerns, but need to be extended to cover missile and weapon mixes and fiscal costs more fully. They need to be explored with a range of parameters to model latency, reconstruction, and added weapons.

The linearity of costs and indices for small forces make it possible to explore the stability characteristics at the goal of current reductions thoroughly. It permits exact optimization of attacks, analytic discussion of the role of survivable forces, and derivation of bilinear stability analyses that clearly delineate the respective roles of missiles and weapons in stability and reconstitution. Since indices reduce to bilinear products of the number of vulnerable missiles and the difference between the normalized second and first strikes there are three ways to produce stability: reduce missiles to zero, reduce $\mathrm{s}-\mathrm{f}$ to zero, or use inaccurate missiles or hidden launchers. It is likely that each will be needed to reach current objectives.

These models can be applied with little modification to conventional or WMD forces, where they reveal a little-discussed problem. The kill probability of nuclear weapons against conventional and WMD forces degrades rapidly with dispersal, so that they become part of the problem rather than of the solution. Since conventional and WMD would be dispersed promptly, that implies urgency in their use while they are still effective. These are problems under current discussion.

Thus, the exchange-stability model derived above captures main features of stability analysis; relates first strike, crisis, and arms control stability as seen from U.S. and Russian perspective; and confirms such lasting questions a whether uncertainty in damage preference or defense deployment can be destabilizing. It illustrates the problems with alternative metrics. It demonstrates the effects of latency and reconstitution in causing the bifurcation of force trajectories at equal damage preference. It shows the main stability issues in deep unilateral and proportional force reductions, and the reconstitution and latency issues at very deep reductions. Applications of this model to conventional and WMD are natural and identify important sensitivities. It is also adequate for the interpretation of current events, policies, and trends. 
Thus, this stability analysis has been effective in pulling together differing analyses, providing a road map of the transition ahead, and identifying connections with related problem areas.

Acknowledgment. The author would like to acknowledge the encouragement and support of E. Arthur and T. Scheber in the execution of this work; P. Kunsberg in its numerical analysis; and P. Goldstone, E. Heighway, M. Yates, and S. Maaranen in discussion of the results. He would also like to thank R. Wagner for stimulating comments on the treatment of latency, V. Reis for comments on results, A. Piontkovsky and E. Velikhov for discussions of first strike metrics and damage preferences, and LtGen G. Kent (USAF Ret.) for discussions of overall exchange models. 


\section{M98004330 \\ ||||||||||||||||||||||||||||||||||||||||||||||||||||||}

Report Number (14) $L A-4 R--97-506 I$

Publ. Date (11) $1997 / 2$
Sponsor Code (18) DOE/DP, XF
UC Category (19) UC-700, DOE/ER 